\title{
Rapid detection of $\alpha$-thalassaemia variants using droplet digital PCR
}

\begin{abstract}
Introduction: Alpha thalassaemia is a highly prevalent disease globally and is a well-known public health problem in Malaysia. The deletional forms of the mutation are the most common forms found in alpha thalassaemia. The three most common deletional alpha thalassaemia found in this region include --SEA deletion, -ŬB.7 rightward and -Ǔ4.2 leftward deletions. The prevalence rate of triplication alpha cases such as ÜŬanti3.7 and ŬŬanti4.2 is not known in Malaysia although it plays a role in exacerbating the clinical phenotypes in beta thalassaemia carriers. Recently, there have been more reported cases of rare alpha thalassaemia mutations due to the advancement of molecular techniques involved in thalassaemia detections. Therefore, it is essential to develop a new method which allows the detection of different alpha thalassaemia mutations including the rare ones simultaneously and accurately.
\end{abstract}

Methods: The purpose of this study was to design an assay for the detection of triplications, common and rare deletional alpha thalassaemia using droplet digital PCR (ddPCR).

Results: This is a quantitative detection method to measure the changes of copy number which can detect deletions, duplications and triplications of the alpha globin gene simultaneously.

Conclusion: In conclusion, ddPCR is an alternative method for rapid detection of alpha thalassaemia variants in Malaysia.

Keyword: ddPCR; Alpha thalassaemia; Detection; Triplication; Malaysia 